\title{
Synthesis of new optically active bis-pyridino- and bis pyridono- 18-crown-6 type ligands containing four lipophilic chains attached to the chiral centers by "two-to-two" type macrocyclization ${ }^{1}$
}

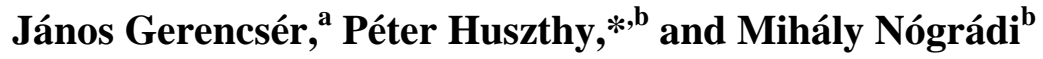 \\ ${ }^{a}$ ComGenex Inc., Budapest, Hungary P.O. Box 73, H-1388 Budapest 62, Hungary \\ ${ }^{b}$ Institute for Organic Chemistry, Budapest University of Technology and Economics and \\ Research Group for Alkaloid Chemistry of the Hungarian Academy of Sciences \\ H-1521 Budapest, Hungary \\ E-mail: huszthy@mail.bme.hu
}

\section{Dedicated to Professor Sándor Antus on his $60^{\text {th }}$ birthday}

(received 05 Sep 03; accepted 30 Oct 03; published on the web 06 Nov 03)

\begin{abstract}
Three new optically active bis-pyridino-18-crown-6 type ligands containing four lipophilic chains at the chiral centers $[(S, S, S, S)-\mathbf{1 0},(R, R, R, R)-\mathbf{1 1}$ and $(S, S, S, S)-12]$ were prepared by sodium ion template effect assisted "two-to-two" type macrocyclizations. The benzyloxysubstituted $(R, R, R, R)-\mathbf{1 1}$ and $(S, S, S, S)-\mathbf{1 2}$ were transformed to the new pyridone analogues $(R, R, R, R)-13$ and $(S, S, S, S)-14$ by catalytic hydrogenation.
\end{abstract}

Keywords: Chiral bis-pyridino- and bis-pyridono-18-crown-6 ligands, "two-to-two" type macrocyclization, template effect, lipophilic crown ethers

\section{Introduction}

To our knowledge only a few optically active bis-pyridino-18-crown-6 type ligands, such as $(R, R, R, R)-1,{ }^{2}(R, R, R, R, R, R, R, R)-2,{ }^{3}(R, R, R, R)-3,{ }^{4,5}$ and $(R, R, R, R)-4$ to $(R, R, R, R)-\mathbf{8},{ }^{5}$ have been prepared (see Figure 1). Among these, the tetramethyl-substituted ligand $(R, R, R, R)-3$ has been studied most. Although it failed to show appreciable enantiomeric selectivity in complexing chiral primary organic ammonium salts, ${ }^{4,6,7}$ ligand $(R, R, R, R)-3$ turned out to be very effective in inducing high enantio- and diastereoselectivity in rare earth metal mediated asymmetric aldol reactions in aqueous media. $5,8,9,10$ 


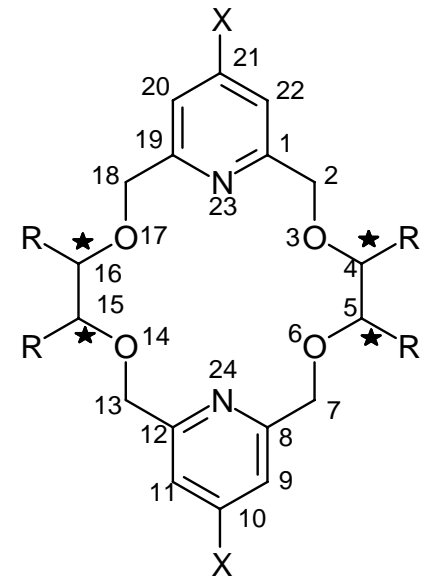

$(R, R, R, R)-1: \mathrm{R}=\mathrm{CONH}_{2}, \quad \mathrm{X}=\mathrm{H}$

$(R, R, R, R, R, R, R, R)-2: \mathrm{R}=\mathrm{C}_{\mathrm{O}}^{\mathrm{O}} \mathrm{X}_{\mathrm{Me}}^{\mathrm{Me}}, \mathrm{X}=\mathrm{H}$

$(R, R, R, R)-3: \mathrm{R}=\mathrm{Me}, \mathrm{X}=\mathrm{H}$

$(R, R, R, R)-4: \mathrm{R}=\mathrm{Et}, \mathrm{X}=\mathrm{H}$

$(R, R, R, R)-5: \mathrm{R}=\mathrm{Ph}, \mathrm{X}=\mathrm{H}$

$(R, R, R, R)-6: \mathrm{R}=\mathrm{Me}, \mathrm{X}=\mathrm{OMe}$

$(R, R, R, R)-7: \mathrm{R}=\mathrm{Me}, \mathrm{X}=\mathrm{Br}$

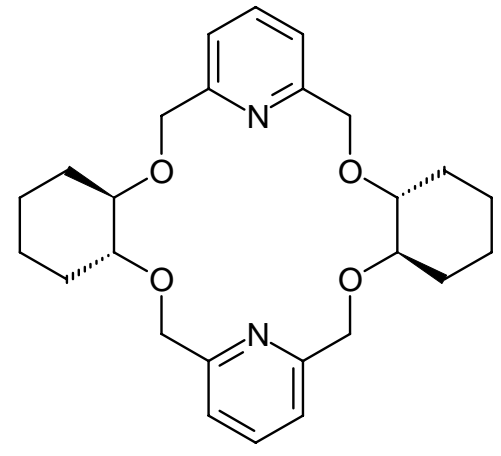

$(R, R, R, R)-8$

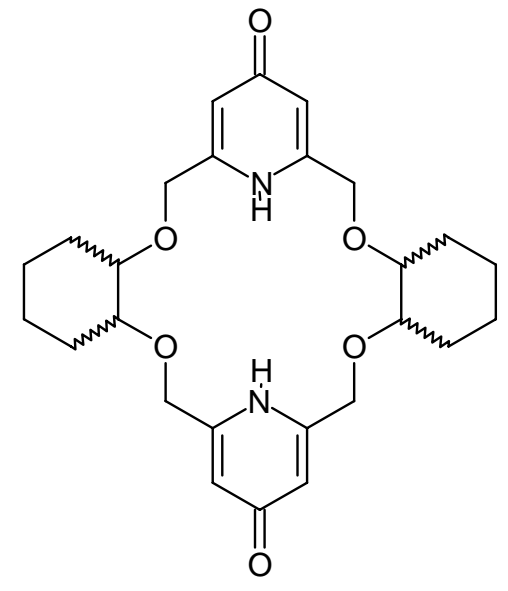

9

Figure 1. Reported optically active bis-pyridino- and bis-p-substituted pyridino-18-crown type ligands and also the reported mixture of racemic- and meso-bis-pyridono-dicyclohexo18-crown-6 macrocycles.

Two procedures for the preparation of $(S, S, S, S)-3$ have been reported. Bradshaw and coworkers prepared $\mathrm{it}^{4}$ in one step from $(2 R, 3 R)$-butane-2,3-diol and 2,6bis(tosyloxymethyl)pyridine by a so called "two-to-two" type sodium ion template assisted cyclization $^{11}$ in $15 \%$ yield. Kobayashi and coworkers ${ }^{5}$ in turn used the same starting materials, but to avoid the low yield of the above method, applied a five-step procedure resulting in an only slightly better overall yield $(18 \%)$. The latter authors extended their procedure to the preparation of the analogues $(R, R, R, R)-\mathbf{4}$ to $(R, R, R, R)-\mathbf{8}$ (see Figure 1) which they obtained in overall yields of $6,1,20,19$ and $9 \%$, respectively. ${ }^{5}$

Our longstanding endeavor has been to prepare optically active lipophilic bis-pyridinoand bis-pyridono-18-crown-6 type macrocycles that would enable the study of the selective transport of metal ions in an aqueous source phase/organic membrane /aqueous receiving phase system $^{12}$ or potentiometric sensing when incorporated into an electrode membrane. ${ }^{13}$ It is known that natural ionophores, such as valinomycin, lasalocid, monensin, etc. also contain several chiral centers and their chirality contributes to the selectivity in complexation and transport of metal ions through biological membranes. ${ }^{14,15}$ Several studies have shown that the stereostructure of chiral synthetic ligands has also a great influence on binding, solvent extraction, and transport of metal ions. ${ }^{15,16,17,18}$ 
Concerning bis-pyridono-18-crown-6 type macrocycles, to our best knowledge only a mixture of racemic- and meso-bis-pyridono-bis-cyclohexano-18-crown-6 ligands 9 (see Figure 1) has been reported. ${ }^{19}$ It was prepared by sodium ion template assisted "two-to-two" type cyclization from racemic trans-cyclohexane-1,2-diol and 4-(tetrahydro-2-pyranyloxy)2,6-bis(tosyloxymethyl)pyridine using sodium hydride in tetrahydrofuran, followed by removal of the protecting group by acid. The overall yield was $8 \%$.

In our search for optically pure chiral macrocycles with enhanced selectivity for metal ions in complexation, solvent extraction, membrane transport and potentiometric studies we prepared a series of new highly lipophilic bis-pyridino ligands, namely (S,S,S,S)-10, the bisp-benzyloxy-substituted crown ethers $(R, R, R, R)-\mathbf{1 1}$ and $(S, S, S, S)-\mathbf{1 2}$ by the "two-to-two" type cyclization method. Removal of the benzyl groups from $(R, R, R, R)-\mathbf{1 1}$ or $(S, S, S, S)-\mathbf{1 2}$ gave bispyridono ligands $(R, R, R, R)-\mathbf{1 3}$ and $(S, S, S, S)-\mathbf{1 4}$ (see. Figure 2).

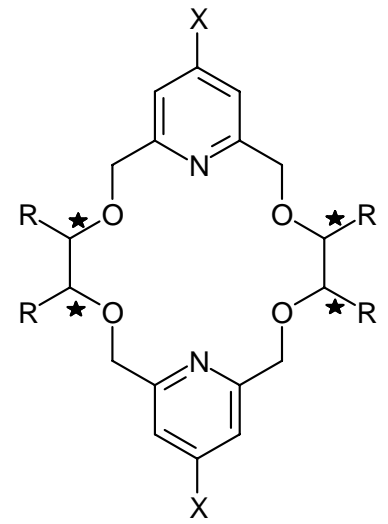

$(S, S, S, S)-10: \mathrm{R}=\mathrm{CH}_{2} \mathrm{OBu}, \mathrm{X}=\mathrm{H}$

$(R, R, R, R)-11: \mathrm{R}=\mathrm{Bu}, \mathrm{X}=\mathrm{OBn}$

$(\mathrm{S}, \mathrm{S}, \mathrm{S}, \mathrm{S})-12: \mathrm{R}=\mathrm{CH}_{2} \mathrm{OBu}, \mathrm{X}=\mathrm{OBn}$

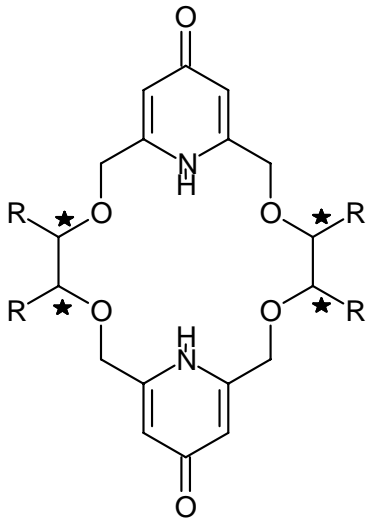<smiles>CCCCOCC1OCCOCCO[C@H](COCCCC)[C@@H](COCCCC)OCCOCCO1</smiles>

$(R, R, R, R)-13: \mathrm{R}=\mathrm{Bu}$

$(S, S, S, S)-14: \mathrm{R}=\mathrm{CH}_{2} \mathrm{OBu}$

Figure 2. New optically active bis-pyridino- bis-p-benzyloxy-substituted-pyridino- and bispyridono-18-crown-6 type ligands and the new tetrabutoxymehtyl-substituted-18-crown-6 ether.

In this paper we also describe the synthesis of the new tetrabutoxymethyl-substituted-18crown-6 ether $(S, S, S, S)$-15 which was performed by the usual "one-to-one" method. We intend to compare the properties of the latter ligand to those containing pyridine, $p$ benzyloxypyridine and pyridone subcyclic units.

The above mentioned studies of the new ligands and also their applicability in stereoselective reactions will be published in the future, here we present only the synthetic work. 


\section{Results and Discussion}

The "two-to-two" type cyclization to $(S, S, S, S)-\mathbf{1 0},(R, R, R, R)-\mathbf{1 1}$, and $(S, S, S, S)-\mathbf{1 2}$ was carried out by forming the disodium salts of the diols $(S, S)-\mathbf{1 6}^{20}$ and $(R, R)-\mathbf{1 7},{ }^{21}$ respectively with sodium hydride in THF followed by the addition of the ditosylates 18 or 19. The template effect of the sodium ion ${ }^{11}$ allowed access to the macrocyclic compounds in yields of 19, 22, and $18 \%$ for $(S, S, S, S)-\mathbf{1 0},(R, R, R, R)-\mathbf{1 1}$ and $(S, S, S, S)-\mathbf{1 2}$, respectively. These yields can be considered as satisfactory for template assisted "two-to-two" type cyclizations. ${ }^{2,3,4,19}$

Preparation of the optically active diols $(S, S)-16^{20}$ and $(R, R)-17,{ }^{21}$ and of the di-p-tosylates $18{ }^{22}$ and $19^{22}$ was performed as reported.

Catalytic hydrogenation of $(R, R, R, R)-\mathbf{1 1}$ and $(S, S, S, S)-12$ gave the bis-pyridono-18-crown6 type macrocycles $(R, R, R, R)-\mathbf{1 3}$ and $(S, S, S, S)-\mathbf{1 4}$, respectively.

Macrocycle $(S, S, S, S)-\mathbf{1 5}$ was obtained by the usual "one-to-one" cyclization from the chiral tetrabutoxymethyl-substituted tetraethylene glycol $(S, S, S, S)-\mathbf{2 0}^{23}$ and bis(2tosyloxyethyl)ether (21) using NaH as a base in THF (Scheme 1).
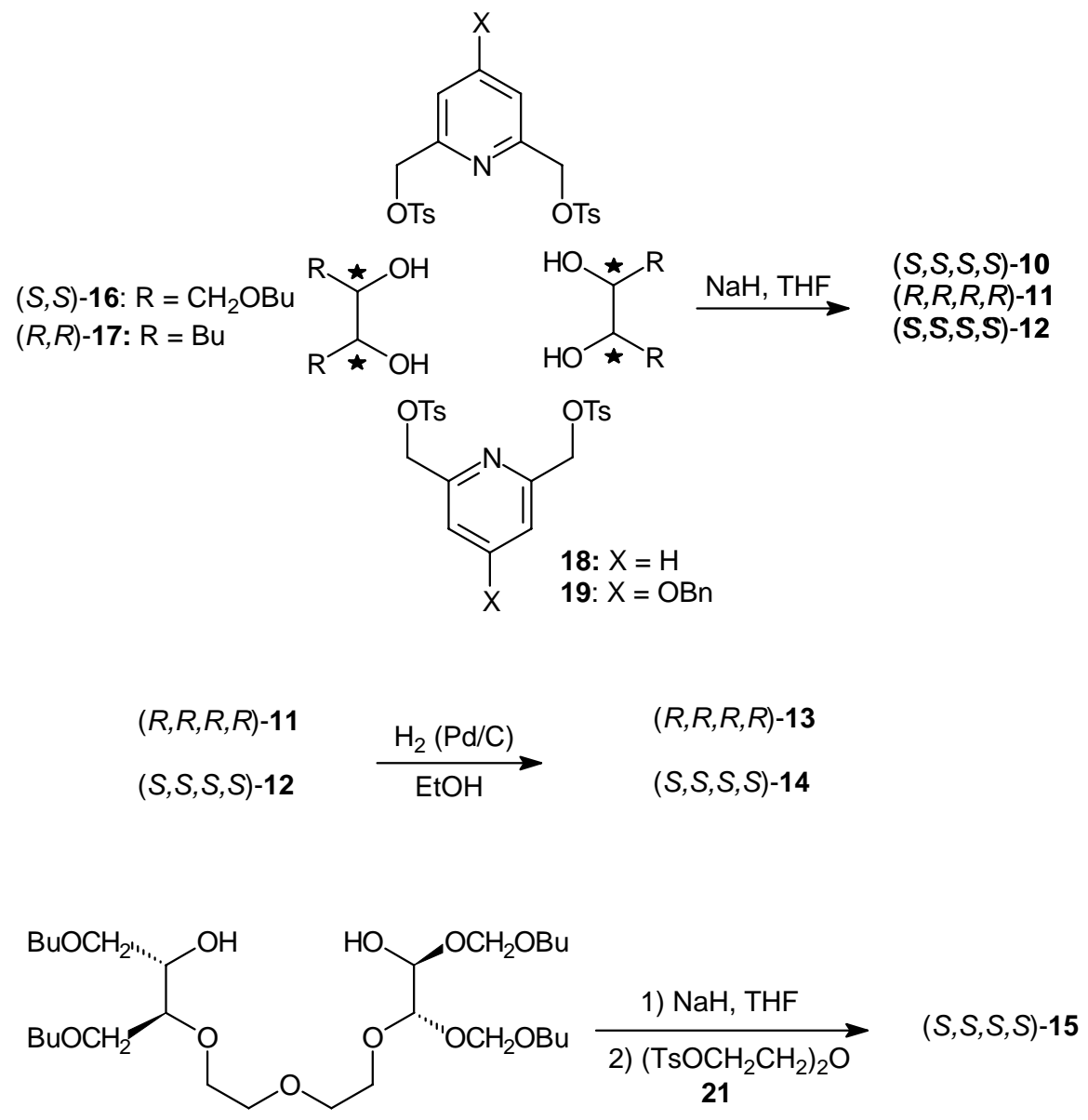

$(S, S, S, S)-20$

Scheme 1. Preparation of the new optically active macrocycles. 


\section{Experimental Section}

General Procedures. Infrared spectra were recorded on a Zeiss Specord IR 75 spectrometer. Optical rotations were taken on a Perkin-Elmer 241 polarimeter that was calibrated by measuring the optical rotations of both enantiomers of menthol. ${ }^{1} \mathrm{H}(500 \mathrm{MHz})$ and ${ }^{13} \mathrm{C}(125$ MHz) NMR spectra were taken on a Bruker DRX-500 Avance spectrometer. Elemental analyses were performed in the Microanalytical Laboratory of the Department of Organic Chemistry, L. Eötvös University, Budapest, Hungary. Molecular weights were determined by a VG-ZAB-2 SEQ reverse geometry mass spectrometer. Melting points were taken on a Boetius micro melting point apparatus and were uncorrected. Starting materials were purchased from Aldrich Chemical Company unless otherwise noted. Silica gel $60 \mathrm{~F}_{254}$ (Merck) and aluminum oxide $60 \mathrm{~F}_{254}$ neutral type $\mathrm{E}$ (Merck) plates were used for TLC. Aluminum oxide (neutral, activated, Brockman I) and silica gel 60 (70-230 mesh, Merck) were used for column chromatography. Romil Ltd. (Cambridge UK) Super Purity Solvent grade THF stored under argon was used in all reactions. Solvents were dried and purified according to the well established methods. Evaporations were carried out under reduced pressure.

\section{(4S,5S,15S,16S)-4,5,15,16-Tetrabutoxymethyl-3,6,14,17-tetraoxa-23,24-diazatricyclo-} [17.3.1.1 $\left.{ }^{8,12}\right]$ tetracosa-1(23),8,10,12(24),19,21-hexaene $[(S, S, S, S)-10]$. A solution of $(S, S)$ $1,4-O, O^{\prime}$-dibutylthreitol $(S, S)-\mathbf{1 6}^{20}(1.50 \mathrm{~g}, 6.4 \mathrm{mmol})$ in THF $(15 \mathrm{~mL})$ was added under argon to a suspension of $\mathrm{NaH}(60 \%$ in mineral oil, $0.72 \mathrm{~g}, 18 \mathrm{mmol})$ in THF $(5 \mathrm{~mL})$. After stirring the reaction mixture under argon for $1 \mathrm{~h}, 2$,6-bis(tosyloxymethyl)pyridine $(18)^{22}(2.86 \mathrm{~g}, 6.4$ mmol) dissolved in THF $(15 \mathrm{~mL})$ was added in one portion at $0{ }^{\circ} \mathrm{C}$. Stirring was continued at r.t. for $16 \mathrm{~h}$, followed by heating under reflux for $24 \mathrm{~h}$. After passing the mixture through a column of $\mathrm{Al}_{2} \mathrm{O}_{3}$ the solution was evaporated and the product purified by chromatography on $\mathrm{SiO}_{2}\left(\mathrm{C}_{6} \mathrm{H}_{6}-\mathrm{EtOH}, 20: 1\right)$ to give $(S, S, S, S)-10,413 \mathrm{mg}(18 \%)$ as an amorphous solid. $[\alpha]_{D}^{23}=$ $+13.4^{\circ}\left(c 0.372, \mathrm{CH}_{2} \mathrm{Cl}_{2}\right) . \delta_{\mathrm{H}}\left(\mathrm{CDCl}_{3}\right)=0.94(\mathrm{t}, J=7.1 \mathrm{~Hz}, 12 \mathrm{H}), 1.33-1.41(\mathrm{~m}, 8 \mathrm{H}), 1.53-$ $1.60(\mathrm{~m}, 8 \mathrm{H}), 3.4-3.6(\mathrm{~m}, 12 \mathrm{H}), 3.66-3.87(\mathrm{~m}, 8 \mathrm{H}), 4.69$ (d, $J=13.2 \mathrm{~Hz}, 4 \mathrm{H}), 4.90(\mathrm{~d}, J=13.2$ $\mathrm{Hz}, 4 \mathrm{H}), 7.37(\mathrm{~d}, J=8.1 \mathrm{~Hz}, 4 \mathrm{H}), 7.66(\mathrm{t}, J=8.1 \mathrm{~Hz}, 2 \mathrm{H}) ; \delta_{\mathrm{C}}\left(\mathrm{CDCl}_{3}\right)=14.1,19.6,32.0$, 70.7, 71.5, 73.4, 78.6, 120.6, 137.1, 157.9; IR (film): $v=3064,3040,2928,2888,2872,1592$, 1540, 1460, 1376, 1312, 1264, 1104, 1004, 992, 856, 804, $760 \mathrm{~cm}^{-1}$; MS (FAB): Calcd. for $\mathrm{C}_{38} \mathrm{H}_{63} \mathrm{~N}_{2} \mathrm{O}_{8}$ 675.4584. Found: $675.4567(\mathrm{M}+\mathrm{H})$.

$(4 R, 5 R, 15 R, 16 R)-10,21-D i b e n z y l o x y-4,5,15,16$-tetrabutyl-3,6,14,17-tetraoxa-23,24diazatri-cyclo[17.3.1.1 $\left.{ }^{8,12}\right]$ tetracosa-1(23),8,10,12(24),19,21-hexaene $\quad[(R, R, R, R)-11] . \quad A$ solution of $(5 R, 6 R)$ - decan-5,6-diol $[(R, R)-17)]^{21}(0.86 \mathrm{~g}, 4.94 \mathrm{mmol})$ in THF $(38 \mathrm{~mL})$ was added to a suspension of $\mathrm{NaH}(60 \%$ in mineral oil, $0.60 \mathrm{~g}, 15 \mathrm{mmol})$ in THF $(12 \mathrm{~mL})$. After stirring the reaction mixture under argon for $1 \mathrm{~h}$, 4-benzyloxy-2,6bis(tosyloxymethyl)pyridine $(\mathbf{1 9})^{22}(2.72 \mathrm{~g}, 4.94 \mathrm{mmol})$ was added in one portion at $0{ }^{\circ} \mathrm{C}$. Stirring was continued at r.t. for $16 \mathrm{~h}$, followed by heating under reflux for $24 \mathrm{~h}$. After 
evaporation of the solvent and addition of water $(20 \mathrm{~mL})$, the mixture was acidified with AcOH to $p H 6$ and extracted with $\mathrm{CH}_{2} \mathrm{Cl}_{2}(30 \mathrm{~mL})$. Evaporation of the solvent and chromatography of the residue on silica gel (hexane-acetone, $4: 1)$ gave $(R, R, R, R)-11$ (420 mg, $22 \%$ ), m.p. $82-83{ }^{\circ} \mathrm{C}(\mathrm{EtOH}) .[\alpha]_{D}^{23}=+3.8^{\circ}\left(\mathrm{c} 0.42, \mathrm{CH}_{2} \mathrm{Cl}_{2}\right) . \delta_{\mathrm{H}}\left(\mathrm{CDCl}_{3}\right)=0.88$ (t, J=7.0 Hz, $12 \mathrm{H}), 1.26-1.27(\mathrm{~m}, 12 \mathrm{H}), 1.37-1.54(\mathrm{~m}, 12 \mathrm{H}), 3.47-3.49(\mathrm{~m}, 4 \mathrm{H}), 4.58(\mathrm{~d}, J=13.0 \mathrm{~Hz}, 4 \mathrm{H})$, $4.66(\mathrm{~d}, J=13.0 \mathrm{~Hz}, 4 \mathrm{H}), 5.06(\mathrm{~s}, 4 \mathrm{H}), 6.88,(\mathrm{~s}, 4 \mathrm{H}), 7.34-7.36(\mathrm{~m}, 10 \mathrm{H}) ; \delta_{\mathrm{C}}\left(\mathrm{CDCl}_{3}\right)=14.1$, $22.7,28.3,29.5,69.5,72.7,79.3,106.6,127.2,127.6,127.9,128.3,159.3$; IR (film): $v=$ 3080, 3030, 2952, 2872, 1600, 1584, 1576, 1496, 1456, 1360, 1344, 1328, 1156, 1136, 1104, 1052, $1020 \mathrm{~cm}^{-1}$; Anal. Calcd. for $\mathrm{C}_{48} \mathrm{H}_{66} \mathrm{~N}_{2} \mathrm{O}_{6}$ (767.1): C, 75.16; H, 8.67; N, 3.65. Found: C, $75.23 ; \mathrm{H}, 8.51 ; \mathrm{N}, 3.42$.

\section{(4S,5S,15S,16S)-10,21-Dibenzyloxy-4,5,15,16-tetrabutoxymethyl-3,6,14,17-tetraoxa-} 23,24-diazatricyclo[17.3.1.1 $\left.{ }^{8,12}\right]$ tetracosa-1(23),8,10,12(24),19,21-hexaene $\quad[(S, S, S, S)-12]$. A solution of diol, $(S, S)-16^{20}(702 \mathrm{mg}, 3 \mathrm{mmol})$ in THF $(10 \mathrm{~mL})$ was added under argon to a suspension of $\mathrm{NaH}(60 \%$ in mineral oil, $340 \mathrm{mg}, 8.5 \mathrm{mmol})$ in THF $(5 \mathrm{~mL})$. After stirring the reaction mixture for $1 \mathrm{~h}$ and heating under reflux for $1 \mathrm{~h}$, di-p-tosylate $(19)^{22}(1.66 \mathrm{~g}, 3 \mathrm{mmol})$ was added in one portion at $-70{ }^{\circ} \mathrm{C}$. After passing the mixture through a column of $\mathrm{Al}_{2} \mathrm{O}_{3}$ the solution was evaporated and the product purified by chromatography on $\mathrm{SiO}_{2}\left(\mathrm{C}_{6} \mathrm{H}_{6}-\mathrm{EtOH}\right.$, 20:1) to give $(S, S, S, S)-12(210 \mathrm{mg}, 18 \%)[\alpha]_{D}^{23}=6.5^{\circ}\left(c 0.34, \mathrm{CH}_{2} \mathrm{Cl}_{2}\right) . \delta_{\mathrm{H}}\left(\mathrm{CDCl}_{3}\right):=0.89(\mathrm{t}$, $J=7.2 \mathrm{~Hz}, 12 \mathrm{H}), 1.36-1.41(\mathrm{~m}, 8 \mathrm{H}), 1.53-1.61(\mathrm{~m}, 8 \mathrm{H}), 3.42-3.54(\mathrm{~m}, 12 \mathrm{H}), 3.68(\mathrm{~d}, J=10.2$ $\mathrm{Hz}, 4 \mathrm{H}), 3.83(\mathrm{~d}, J=6.2 \mathrm{~Hz}, 4 \mathrm{H}), 4.63(\mathrm{~s}, 8 \mathrm{H}), 5.08(\mathrm{~s}, 4 \mathrm{H}), 7.00(\mathrm{~s}, 4 \mathrm{H}), 7.30-7.42(\mathrm{~m}, 10 \mathrm{H})$; $\delta_{\mathrm{C}}\left(\mathrm{CDCl}_{3}\right):=14.1,19.6,32.0,70.0,70.7,71.6,73.1,78.5,107.2,127.9,128.5,128.8,136.1$, 160.4, 166.4; Anal. Calcd. for $\mathrm{C}_{52} \mathrm{H}_{74} \mathrm{~N}_{2} \mathrm{O}_{10}$ (887.1): C, 70.40; H, 8.41; N, 3.16. Found: C, 70.32; H, 8.48; N, 3.24; IR (film): $v=3080,3062$, 3030, 2958, 2890, 2872, 1602, 1582, 1573, $1498,14581364,1328,1155,1136,1102,1052,1020 \mathrm{~cm}^{-1}$.

\section{$(4 R, 5 R, 15 R, 16 R)-4,5,15,16-T e t r a b u t y l-3,6,14,17-t e t r a o x a-23,24-$}

\section{diazatricyclo[17.3.1.1 $\left.{ }^{8,12}\right]$-tetracosa1(22),8,11,19-tetraene-10,21(23H,24H)-dione}

[( $R, \boldsymbol{R}, \boldsymbol{R}, \boldsymbol{R})-13] .(R, R, R, R)-11(160 \mathrm{mg}, 0.21 \mathrm{mmol})$ was hydrogenated in EtOH $(10 \mathrm{~mL})$ over palladium-on-charcoal catalyst (30 $\mathrm{mg}$ ). The usual work-up and trituration of the residue with hot acetone gave the product (100 mg, 77\%), m.p. $241-243{ }^{\circ} \mathrm{C} .[\alpha]_{D}^{23}=-5.7^{\circ}\left(c 0.52, \mathrm{CH}_{2} \mathrm{Cl}_{2}\right)$. ${ }^{1} \mathrm{H} \mathrm{NMR}\left(\mathrm{CDCl}_{3}+\mathrm{CF}_{3} \mathrm{CO}_{2} \mathrm{H}\right): \delta=0.86-0.91(\mathrm{~m}, 12 \mathrm{H}), 1.26-1.34(\mathrm{~m}, 24 \mathrm{H}), 3.62(\mathrm{~m}, 4 \mathrm{H}), 4.79$ $(\mathrm{d}, J=15.0 \mathrm{~Hz}, 4 \mathrm{H}), 4.98(\mathrm{~d}, J=15.0 \mathrm{~Hz}, 4 \mathrm{H}), 7.07(\mathrm{~s}, 4 \mathrm{H}), 11.67(\mathrm{~s}, 2 \mathrm{H}), \delta_{\mathrm{C}}\left(\mathrm{CDCl}_{3}+\right.$ $\mathrm{CF}_{3} \mathrm{CO}_{2} \mathrm{H}$ ): $=13.7,22.6,27.5,30.5,67.4,83.0$, 109.9, 152.1, 171.6; MS (FAB): Calcd. for $\mathrm{C}_{34} \mathrm{H}_{55} \mathrm{~N}_{2} \mathrm{O}_{6}$ 587.4060. Found: $587.4062(\mathrm{M}+\mathrm{H})$; IR (KBr): $v=3432,3224,2950,2886,2872$, $1636,1558,1524,1452,1432,1408,1350,1100,952,858 \mathrm{~cm}^{-1}$.

$(4 R, 5 R, 15 R, 16 R)-4,5,15,16$-Tetrabutoxymethyl-3,6,14,17-tetraoxa-23,24-diazatricyclo[17.3.1.1 $\left.{ }^{8,12}\right]$-tetracosa-1(22),8,10,11,18-tetraene-10(23H),21(24H)-dione $[(S, S, S, S)-14]$

$(S, S, S, S)-12$ (93 mg, $0.105 \mathrm{mmol})$ was hydrogenated in EtOH as described for $(R, R, R, R)-13$. The usual work-up and purification by TLC gave the product (55 $\mathrm{mg}, 74 \%$ ) as a waxy solid. $[\alpha]_{D}^{23}=+26.6^{\circ}\left(c 1.08, \mathrm{CH}_{2} \mathrm{Cl}_{2}\right) ; \delta_{\mathrm{H}}\left(\mathrm{CDCl}_{3}\right):=0.89(\mathrm{t}, J=7.2 \mathrm{~Hz}, 12 \mathrm{H}), 1.30-1.33(\mathrm{~m}, 8 \mathrm{H})$, $1.48-1.54(\mathrm{~m}, 8 \mathrm{H}), 3.36-3.43(\mathrm{~m}, 8 \mathrm{H}), 3.37-3.41(\mathrm{~m}, 4 \mathrm{H}), 3.59-3.61(\mathrm{~m}, 4 \mathrm{H}), 4.59(\mathrm{~d}, J=14.0$ 
$\mathrm{Hz}, 4 \mathrm{H}), 4.73(\mathrm{~d}, J=14.0 \mathrm{~Hz}, 4 \mathrm{H}), 6.32(\mathrm{~s}, 4 \mathrm{H}) ; \delta_{\mathrm{C}}\left(\mathrm{CDCl}_{3}\right):=13.8,19.3,31.5,68.5,69.7$, 71.6, 78.8, 113.6, 147.9, 169.0; $\mathrm{MS}$ (FAB): Calcd. for $\mathrm{C}_{38} \mathrm{H}_{63} \mathrm{~N}_{2} \mathrm{O}_{10}$ 707.4483. Found: $707.4486(\mathrm{M}+\mathrm{H})$;. IR (film): $v=3432,3128,2960,2928,2870,1640,1488,1424,1384$, $1204,1116 \mathrm{~cm}^{-1}$.

(2S,3S,11S,12S)-2,3,11,12-Tetrabutoxymethyl-18-crown-6 [(S,S,S,S)-15] $(7 S, 8 S, 16 S, 17 S)$ 8,16-bis(butoxymethyl)-5,9,12,15,19-pentaoxatricosane-7-17-diol $\left[(S, S, S, S)-2^{23}\right](538 \mathrm{mg}$, $1.00 \mathrm{mM}$ ) and bis(2-tosyloxyethyl)ether (21) (414 mg, $1.00 \mathrm{mM})$ was converted to $(S, S, S, S)$ 15 (318 mg, $52 \%$ ) as described above for compound $(S, S, S, S)-10 .[\alpha]_{D}^{25}=+13.8^{\circ}$ (c 0.76 , $\left.\mathrm{CH}_{2} \mathrm{Cl}_{2}\right) .{ }^{1} \mathrm{H}$ NMR $\left(\mathrm{CDCl}_{3}\right): \delta=0.91(\mathrm{t}, J=7.2 \mathrm{~Hz}, 12 \mathrm{H}), 1.33-1.40(\mathrm{~m}, 8 \mathrm{H}), 1.52-1.57(\mathrm{~m}$, $8 \mathrm{H}), 3.37-3.46(\mathrm{~m}, 12 \mathrm{H}), 3.57-3.64(\mathrm{~m}, 20 \mathrm{H}), 3.79-3.85(\mathrm{~m}, 4 \mathrm{H}) ;{ }^{13} \mathrm{C} \mathrm{NMR}\left(\mathrm{CDCl}_{3}\right): \delta=14.4$ 19.6, 32.0, 70.9, 71.3, 71.6, 71.7, 80.1; MS (FAB) Calcd. For $\mathrm{C}_{32} \mathrm{H}_{64} \mathrm{O}_{10} \mathrm{Na}$ 631.4901. Found $631.4923(\mathrm{M}+\mathrm{Na})$. IR (film) $v_{\max }=2928,2896,2864,1464,1376,1320,1296,1248,1120$, $844 \mathrm{~cm}^{-1}$.

\section{Acknowledgements}

Financial support of the Hungarian National Science Fund (OTKA grant T-038393) is gratefully acknowledged.

\section{References}

1. Presented at the XXVII International Symposium on Macrocyclic Chemistry, June 23-27, 2002, Park City (Utah, USA).

2. Girodeau, J. M.; Lehn, J. M.; Sauvage, J. P. Angew. Chem. 1975, 87, 813.

3. Laidler, D. A.; Stoddart, J. F. J. Chem. Soc., Chem. Commun. 1976, 979.

4. Bradshaw, J. S.; Huszthy, P.; McDaniel, C. W.; Zhu, C. Y.; Dalley, N. K.; Izatt, R. M.; Lifson, S. J. Org. Chem. 1990, 55, 3129.

5. Hamada, T.; Manabe, K.; Ishikawa, S.; Nagayama, S.; Shiro, M.; Kobayashi, S. J. Am. Chem. Soc. 2003, 125, 2989.

6. Izatt, R. M.; Zhu, C. Y.; Huszthy, P.; Bradshaw, J. S. In Enantiomeric Recognition in Macrocyclic-Primary Ammonium Cation Sytems in Crown Ethers: Toward Future Applications; Cooper, S. R., Ed.; VCH: New York, 1992; p 207.

7. Zhang, X. X.; Izatt, R. M.; Bradshaw, J. S.; Huszthy, P. Anales de Quiímica Int. Ed. 1996, 92, 64.

8. Kobayashi, S.; Hamada, T.; Nagayama, S.; Manabe, K. Org. Lett. 2001, 3, 165.

9. Kobayashi, S.; Hamada, T.; Nagayama, S.; Manabe, K. J. Brazilian Chem. Soc. 2001, 12, 627.

10. Kobayashi, S. Manabe, K. Acc. Chem. Res. 2002, 35, 209. 
11. Steed, J. W.; Atwood, J. K. Supramolecular Chemistry, John Wiley and Sons Ltd.: Chichester, 2000, p125.

12. Izatt, R. M.; LindH, G. C.; Bruening, R. L. Huszthy, P.; McDaniel, C. W.; Bradshaw, J. S.; Christensen, J. J. Anal. Chem. 1988, 60, 1694.

13. Horváth, V.; Takács, T.; Horvai, Gy.; Huszthy, P.; Bradshaw, J. S. Izatt, R. M. Anal. Lett. 1997, 30, 1591.

14. Gokel, G. W.; Nakano, A. In Feeble Forces and Flexible Frameworks in Crown Ethers: Toward Future Applications; Cooper, S. R. Ed.; VCH: New York, 1992; p1.

15. Tsukube, H.; Yamada, T.; Shinoda, S. Ind. Eng. Chem. Res. 2000, 39, 3412.

16. Erickson, S. D.; Still, W. C. Tetrahedron Lett. 1990, 31, 4243.

17. Sasaki, S.; Naito, H.; Maruta, K.; Kawahara, E.; Maeda, M. Tetrahedron Lett. 1994, 35, 3337.

18. Shibutani, Y.; Mino, S.; Long, S. S.; Moriuchi-Kawakami, T.; Yakabe, K.; Shono, T. Chem. Lett. 1997, 49.

19. Bradshaw, J. S.; Krakowiak, K. E.; Huszthy, P.; Izatt, R. M. J. Heterocyclic Chem. 1991, 28, 773.

20. Seebach, D.; Kalinowski, H.-O.; Bastani, B.; Crass, G.; Daum, H.; Dörr, H.; DuPreez, N. P.; Ehrig, V.; Langer, W.; Nüssler, C.; Oei, H.-A.; Schmidt, M. Helv. Chim. Acta 1977, 60, 301.

21. Sharpless, K. B; Amberg, W.; Beller, M; Chen, H.; Hartung, J.; Kawanami, Y.; Lübben, D.; Manoury, E.; Ogino, Y.; Shibata, T.; Ukita: T. J. Org. Chem. 1991, 56, 4585.

22. Horváth, G.; Rusa, C.; Köntös, Z.; Gerencsér, J.; Huszthy, P. Synth. Comm. 1999, 29, 3719.

23. Gerencsér, J.; Báthori, N.; Czugler, M.; Huszthy, P.; Nógrádi, M. Tetrahedron Asymmetry 2003, 14, 2803.

24. Riddick, J. A.; Burger, W. B. Organic Solvents in Techniques of Organic Chemistry, $3^{\text {rd }}$ Edn, Weissberg, A. Ed.; Wiley-Interscience: New York, 1970; Vol II. 